\title{
High resolution entropy stable scheme for shallow water equations
}

\author{
Xiaohan Cheng ${ }^{1, \mathrm{a}}$, Yufeng $\mathrm{Nie}^{2, \mathrm{~b}}$ \\ ${ }^{1,2}$ Department of Applied Mathematics, Northwestern Polytechnical \\ University, \\ Xi'an 710072, Shaanxi, China \\ achengxh168@163.com, ${ }^{b} y$ fnie@nwpu.edu.cn
}

\begin{abstract}
:
A high resolution entropy stable scheme is proposed for solving shallow water equations in this paper. The scheme contains two parts: entropy conservative flux and numerical diffusion operator. To achieve high resolution, a limiter is employed to guarantee the numerical diffusion term being added around the discontinuities automatically. Numerical experiments are presented to demonstrate the proposed scheme's capacity.
\end{abstract}

Keywords: entropy stable scheme, limiter, shallow water equations, high resolution

\section{Introduction}

Shallow water equations, also known as the Saint-Venant system, are widely used to model flows in rivers, lakes and near-shore oceans. In one dimensional case the equations can be written as a system of balance laws

$$
U_{t}+f(U)_{x}=-s(x, U),
$$

with $\quad U=[h, h u]^{T}$ being the conservative vector, $f=\left[h u, h u^{2}+\frac{1}{2} g h^{2}\right]^{T}$ being the flux vector, and $s=\left[0, g h b_{x}\right]^{T}$ being the source term. Here, $b(x)$ denotes the bottom topography, $h(x, t)$ is the water 
height above the bottom and $u(x, t)$ is the depth-averaged water velocity. The constant $g$ is the gravity acceleration. In Eq. (1), only the geometrical source term is considered. Other effects, such as wind forces and friction on the surface, are neglected.

The most striking feature of balance laws, as well as conservation laws, is that solutions with discontinuities may appear in a finite time even for sufficient smooth initial data [1]. Thus, solutions are in general sought in the weak sense. Due to the non-uniqueness of weak solutions, an additional criterion, termed entropy condition, must be imposed to select the physical solution of our interest. Numerical schemes which respect a discrete version of the entropy dissipation statement were called entropy stable schemes.

Entropy stable scheme was first presented by Tadmor [2] and has attracted much attention in recent years $[3,4,5]$. Most of them were based on entropy conservative flux and suitable numerical diffusion operator. Entropy conservative flux preserves the entropy exactly and behaves well in smooth regions. However, spurious oscillation will be produced around the discontinuities, such as shocks. Thus, some dissipative mechanism should be contained to achieve entropy stability. Compared with the popular used Weighted Essentially Non-Oscillatory (WENO), Discontinuous Galerkin (DG) methods [6,7], entropy stable schemes satisfy the system's additional condition, i.e. entropy inequality, and can avoid some unphysical phenomena. These methods are promising when solving realistic engineering problems.

In this paper, a high resolution entropy stable method is developed to solve shallow water equations. The scheme consists of a second order entropy conservative flux and a Roe-type numerical dissipation term. Unlike the approach in [8], numerical dissipation term is activated on the whole the whole domain and lowers the accuracy in smooth areas. Although a construction of second order accurate is performed to avoid this, the resulting scheme cannot be entropy stable. Here, a limiter, which signals the smooth degree of solutions, is employed to make the numerical dissipation work around the discontinuities automatically. By this way, the obtained scheme still guarantees entropy stability. 
When handing the source term, the well balance property is also taken into account. Finally, numerical examples are presented to verify how well the scheme performs in practice.

\section{Numerical method}

For simplicity, uniform grids of size $\Delta x$ are considered. Then, a standard semi-discrete finite volume scheme is given by

$$
\frac{d}{d t} U_{i}=-\frac{1}{\Delta x}\left(F_{i+1 / 2}-F_{i-1 / 2}\right)-s_{i} .
$$

Here, $U_{i}$ is the cell average on $I_{i}=\left[x_{i-1 / 2}, x_{i+1 / 2}\right], F_{i+1 / 2}$ is the numerical flux consistent with the flux $f$ and $s_{i}$ is a discretized source term. Time integration is carried out with a second order strong stability preserving (SSP) Runge-Kutta method [9]: given a solution $U_{i}^{n}$ at time step $t_{n}$, the solution at the next time level $U_{i}^{n+1}$ is advanced by

$$
\left\{\begin{array}{l}
U_{i}^{*}=U_{i}^{n}+\Delta t^{n} L\left(U_{i}^{n}\right), \\
U_{i}^{n+1}=\frac{1}{2}\left(U_{i}^{n}+U_{i}^{*}+\Delta t^{n} L\left(U_{i}^{*}\right)\right),
\end{array}\right.
$$

where $L$ is the right-hand side of (2).

Shallow water system possesses an entropy function $E(U)=\frac{1}{2}\left(h u^{2}+g h^{2}+g h b\right)$ and the entropy variables are defined by $V=\frac{\partial E}{\partial U}=\left[g(h+b)-\frac{u^{2}}{2}, u\right]^{T}$. Then, the corresponding entropy flux functions are given by $Q(U)=\frac{1}{2} h u^{3}+g h^{2} u \quad$ and 
$J(x, U)=g h b u$, respectively. Define the entropy potential as $\psi=V^{T} f-Q=\frac{1}{2} g u h^{2}$. According to the lemma in [8], a numerical flux $F_{i+1 / 2}^{E C}$ is entropy conservative if

$$
\square V_{i+1 / 2}^{T} \square F_{i+1 / 2}^{E C}=\square \psi \bigsqcup_{i+1 / 2}+g \square b \bigsqcup_{i+1 / 2} \bar{h}_{i+1 / 2} \bar{u}_{i+1 / 2},
$$

with the notation: $\square a \bigsqcup_{i+1 / 2}=a_{i+1}-a_{i}, \bar{a}_{i+1 / 2}=\frac{1}{2}\left(a_{i}+a_{i+1}\right)$. Then, analogous to the case with flat bottom topography, $F_{i+1 / 2}^{E C}$ is determined by

$$
F_{i+1 / 2}^{E C}=\left[\begin{array}{l}
\bar{h}_{i+1 / 2} \bar{u}_{i+1 / 2} \\
\frac{g}{2} \bar{h}^{2}{ }_{i+1 / 2}+\bar{h}_{i+1 / 2}\left(\bar{u}_{i+1 / 2}\right)
\end{array}\right] .
$$

To achieve the well balanced property, the source term is approximated by

$$
S_{i}=\left[\begin{array}{l}
0 \\
\frac{g}{2 \Delta x}\left(\bar{h}_{i+1 / 2} \square b \bigsqcup_{i+1 / 2}+\bar{h}_{i-1 / 2} \square b \bigsqcup_{i-1 / 2}\right)
\end{array}\right] .
$$

Entropy conservative flux preserves the total entropy and performs well in smooth regions. But it will produce spurious oscillations in the vicinity of discontinuities. This problem is handled by using a suitable numerical operator. Consider the following numerical flux,

$$
F_{i+1 / 2}^{E S}=F_{i+1 / 2}^{E C}-\frac{1}{2} R_{i+1 / 2} \Phi_{i+1 / 2} \Lambda_{i+1 / 2} R_{i+1 / 2}^{T} \square V \bigsqcup_{i+1 / 2},
$$

with 


$$
\begin{aligned}
& R_{i+1 / 2}=\frac{1}{\sqrt{2 g}}\left[\begin{array}{cc}
1 & 1 \\
\bar{u}_{i+1 / 2}-\sqrt{g \bar{h}_{i+1 / 2}} & \bar{u}_{i+1 / 2}+\sqrt{g \bar{h}_{i+1 / 2}}
\end{array}\right], \\
& \Lambda_{i+1 / 2}=\operatorname{diag}\left(\left|\bar{u}_{i+1 / 2}-\sqrt{g \bar{h}_{i+1 / 2}}\right|,\left|\bar{u}_{i+1 / 2}+\sqrt{g \bar{h}_{i+1 / 2}}\right|\right), \\
& \Phi_{i+1 / 2}=\operatorname{diag}\left(1-\phi_{i+1 / 2}^{1}, 1-\phi_{i+1 / 2}^{2}\right) .
\end{aligned}
$$

The parameter $\phi_{i+1 / 2}$ is computed by the Combined Superbee limiter[10].

We want to stress here that the above defined numerical flux is entropy stable. The introduced $\Phi$ possesses a property of being close to 1 at shocks and vanishing away from shocks so that the numerical diffusion works around discontinuities automatically.

\section{Numerical experiments}

In all simulations below, the gravitational constant is fixed to be $g=1$ and Neumann boundary conditions are imposed. The reference solutions are computed from a well balanced second order central upwind scheme from [11] on a mesh of 2000 points.

First, small perturbation of a steady state solution is considered. The bottom contains a hump,

$$
b(x)= \begin{cases}0.25(\cos (10 \pi(x-0.5))+1), & |x-0.5|<0.1 \\ 0, & \text { otherwise }\end{cases}
$$

The initial data is set to be $(h+b)_{x, t=0}=1+\varepsilon$ and $u_{x, t=0}=0$. The perturbation constant $\varepsilon$ equals to 0.01 for $0.1<x<0.2$. This example is simulated on a mesh of 200 grids and the water surface level $h+b$ at time $t=0.7$ is presented in Fig. 1 . It is seen that the scheme resolves the wave accurately without numerical oscillations. 


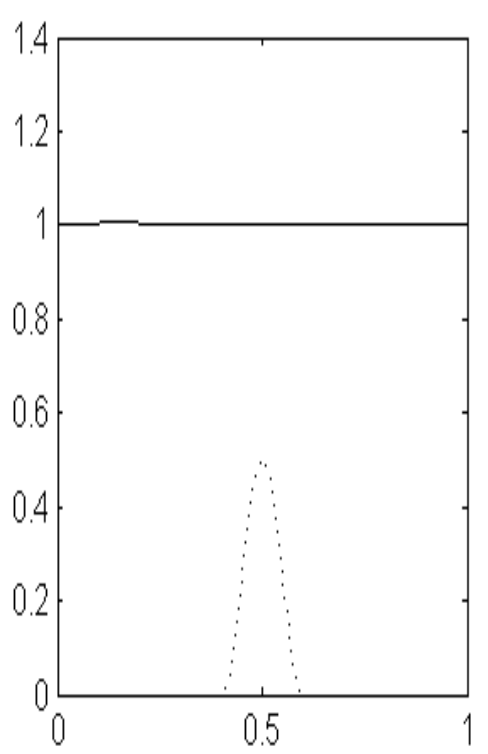

(a)

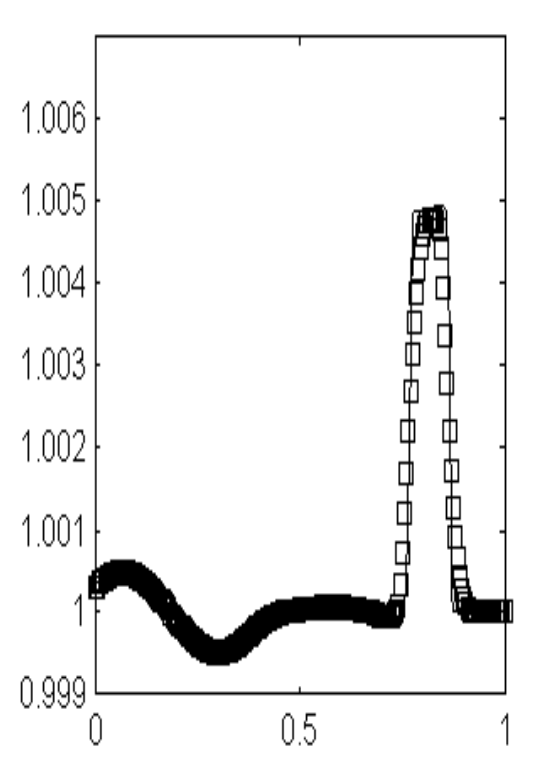

(b)

Fig. 1. Small perturbation of a steady state solution. (a) Initial water surface level $h+b$ (solid line) and bottom topography (dotted line); (b) Water surface level $h+b$ at $t=0.7$ (square: numerical solution; solid line: reference solution)

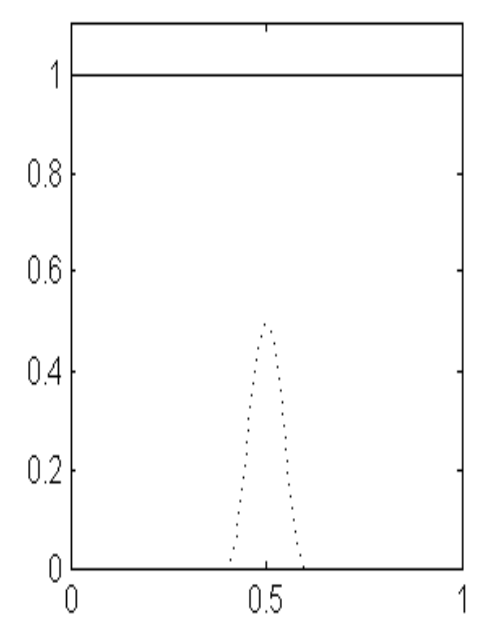

(a)

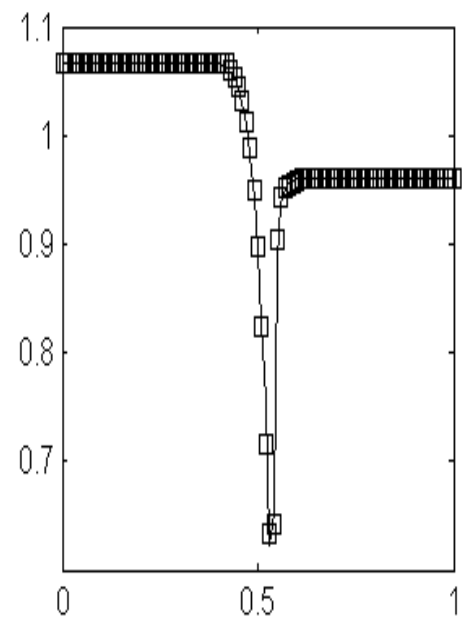

(b)

Fig. 2. A stationary shock in the transcritical case. (a) Initial water surface level $h+b$ (solid line) and bottom topography (dotted line); (b) Water surface level $h+b$ at $t=1.8$ (square: numerical solution; solid line: reference solution) 
Next, a transcritical flow is simulated. The bottom topography is the same as the previous example. The initial water surface level is set to be 1 and the initial water velocity is taken to be 0.3 . In this example, a steady-state appears on the surface. The computation is performed on a mesh of 100 grids up to time $t=1.8$ and Fig. 2 displays the water surface level. Clearly, the shock is fully captured.

\section{Conclusions}

In this paper, a high resolution entropy stable method has been developed for simulating shallow water flows. Its principal advantage, and a major difference from other existing methods, is that it achieves entropy stability. The scheme's accuracy is improved by using a limiter. Numerical simulations demonstrate the proposed scheme is efficient and high resolution.

\section{Acknowledgements}

This research was financially supported by the National Natural Science Foundation of China $(11171043,11471261)$ and the Doctorate Foundation of Northwestern Polytechnical University (CX201426).

\section{References}

[1]. RJ LeVeque, Finite volume methods for hyperbolic problems. Vol. 31. 2002: Cambridge university press.

[2]. E Tadmor. Math. Comp., 1987, 49(179):91-103.

[3]. E Tadmor. Acta Numer., 2003, 12(1):451-512.

[4]. US Fjordholm, S Mishra, and E Tadmor. SIAM J. Numer. Anal., 2012, 50(2):544-573.

[5]. A Hiltebrand and S Mishra. Numer. Math., 2014, 126(1):103-151.

[6]. C-W Shu. SIAM Rev., 2009, 51(1):82-126.

[7]. B Cockburn and C-W Shu. J. Sci. Comput., 2001, 16(3):173-261.

[8]. US Fjordholm, S Mishra, and E Tadmor. J. Comput. Phys., 2011, 
230(14):5587-5609.

[9]. S Gottlieb, DI Ketcheson, and C-W Shu. J. Sci. Comput., 2009, 38(3):251-289.

[10]. PK Sweby. SIAM J. Numer. Anal., 1984, 21(5):995-1011.

[11]. A Kurganov and D Levy. ESAIM: Math. Modell. Numer. Anal., 2002, 36(3):397-425. 